UCRHEP-T429

July 2007

\title{
New Lepton Family Symmetry and Neutrino Tribimaximal Mixing
}

\author{
Ernest Ma \\ Physics and Astronomy Department \\ University of California, Riverside \\ Riverside, California 92521, USA
}

\begin{abstract}
The newly proposed finite symmetry $\Sigma(81)$ is applied to the problem of neutrino tribimaximal mixing. The result is more satisfactory than those of previous models based on $A_{4}$ in that the use of auxiliary symmetries (or mechanisms) may be avoided. Deviations from the tribimaximal pattern are expected, but because of its basic structure, only $\tan ^{2} \theta_{12}$ may differ significantly from 0.5 (say 0.45 ) with $\sin ^{2} 2 \theta_{23}$ remaining very close to one, and $\theta_{13}$ very nearly zero.
\end{abstract}


Based on present neutrino-oscillation data, the neutrino mixing matrix $U_{\alpha i}$ linking the charged leptons $(\alpha=e, \mu, \tau)$ to the neutrino mass eigenstates $(i=1,2,3)$ is determined to a large extent [1]. In particular, a good approximate description is that of the so-called tribimaximal mixing of Harrison, Perkins, and Scott [2], i.e.

$$
U_{\alpha i}=\left(\begin{array}{ccc}
\sqrt{2 / 3} & \sqrt{1 / 3} & 0 \\
-\sqrt{1 / 6} & \sqrt{1 / 3} & -\sqrt{1 / 2} \\
-\sqrt{1 / 6} & \sqrt{1 / 3} & \sqrt{1 / 2}
\end{array}\right) .
$$

Using the discrete lepton family symmetry group $A_{4}[3$, 4], this pattern has been discussed in a number of recent papers with varying additional assumptions [5, 6, 7, 8, 9, 10, 11, 12, 13, 14, 15, 16]. In particular, auxiliary symmetries (or mechanisms) beyond $A_{4}$ are required to enforce the following conflicting alignment of vacuum expectation values: $(1,1,1)$ for a $\mathbf{3}$ representation which couples to charged leptons, and $(1,0,0)$ for a $\mathbf{3}$ representation which couples to neutrinos. As shown below, this problem may be alleviated if $A_{4}$ is replaced by another finite discrete symmetry $\Sigma(81)$, which was recently proposed [17].

Consider the basis $\left(a_{1}, a_{2}, a_{3}\right)$ and the $Z_{3}$ transformation

$$
a_{1} \rightarrow a_{2} \rightarrow a_{3} \rightarrow a_{1}
$$

If this is supplemented with the $Z_{2}$ transformation

$$
a_{1,2} \rightarrow-a_{1,2}, \quad a_{3} \rightarrow a_{3}
$$

then the group generated is $A_{4}$, which is the symmetry group of the even permutation of 4 objects, and that of the perfect tetrahedron [18]. It is also a subgroup of $S U(3)$, denoted as $\Delta(12)$. If Eq. (2) is supplemented instead with another $Z_{3}$, i.e.

$$
a_{1} \rightarrow a_{1}, \quad a_{2} \rightarrow \omega a_{2}, \quad a_{3} \rightarrow \omega^{2} a_{3},
$$

where $\omega=\exp (2 \pi i / 3)=-1 / 2+i \sqrt{3} / 2$, then the group generated is $\Delta(27)$ [19, 20, 21], which is also a subgroup of $S U(3)$. If Eq. (4) is replaced with

$$
a_{1} \rightarrow \omega a_{1}, \quad a_{2,3} \rightarrow a_{2,3}
$$


then the group generated, call it $\Sigma(81)$, contains $\Delta(27)$. It is a subgroup of $U(3)$ but not $S U(3)$. [Note that Eqs. (2) and (5) are sufficient for generating the four transformations used to obtain $\Sigma(81)$ in Ref. [17].] It has 9 one-dimensional irreducible representations $\mathbf{1}_{\mathbf{i}}(i=1, \ldots, 9)$ and 8 three-dimensional ones $\mathbf{3}_{\mathbf{A}}, \overline{\mathbf{3}}_{\mathbf{A}}, \mathbf{3}_{\mathbf{B}}, \overline{\mathbf{3}}_{\mathbf{B}}, \mathbf{3}_{\mathbf{C}}, \overline{\mathbf{3}}_{\mathbf{C}}, \mathbf{3}_{\mathbf{D}}, \overline{\mathbf{3}}_{\mathbf{D}} . \quad$ Its $17 \times 17$ character table and the 81 matrices of its defining representation $\mathbf{3}_{\mathbf{A}}$ are given in Ref. [17].

Consider the supersymmetric extension of the Standard Model with 3 lepton families. Under $\Sigma(81)$, let

$$
\begin{aligned}
& L_{i}=\left(\nu_{i}, l_{i}\right) \sim \mathbf{3}_{\mathbf{A}}, \quad l_{i}^{c} \sim \mathbf{1}_{\mathbf{1}, \mathbf{2}, \mathbf{3}}, \quad \Phi=\left(\phi^{0}, \phi^{-}\right) \sim \mathbf{1}_{\mathbf{1}}, \\
& \sigma_{i} \sim \mathbf{3}_{\mathbf{A}}, \quad \bar{\sigma}_{i} \sim \overline{\mathbf{3}}_{\mathbf{A}}, \quad \chi_{i} \sim \mathbf{3}_{\mathbf{B}}, \quad \bar{\chi}_{i} \sim \overline{\mathbf{3}}_{\mathbf{B}}, \quad \xi=\left(\xi^{++}, \xi^{+}, \xi^{0}\right) \sim \mathbf{1}_{\mathbf{1}} .
\end{aligned}
$$

Using the multiplication rules given in the Appendix, the allowed quadrilinear Yukawa terms are $\left(L_{1} \bar{\sigma}_{1}+L_{2} \bar{\sigma}_{2}+L_{3} \bar{\sigma}_{3}\right) l_{1}^{c} \Phi,\left(L_{1} \bar{\sigma}_{1}+\omega^{2} L_{2} \bar{\sigma}_{2}+\omega L_{3} \bar{\sigma}_{3}\right) l_{2}^{c} \Phi,\left(L_{1} \bar{\sigma}_{1}+\omega L_{2} \bar{\sigma}_{2}+\omega^{2} L_{3} \bar{\sigma}_{3}\right) l_{3}^{c} \Phi$, $\left(L_{1} L_{1} \sigma_{1}+L_{2} L_{2} \sigma_{2}+L_{3} L_{3} \sigma_{3}\right) \xi$, and $\left(L_{1} L_{2} \chi_{3}+L_{2} L_{3} \chi_{1}+L_{3} L_{1} \chi_{2}\right) \xi$. As shown below, the singlet superfields $\sigma_{i}, \bar{\sigma}_{i}, \chi_{i}, \bar{\chi}_{i}$ will acquire vacuum expectation values without breaking the supersymmetry. The desirable solutions $(1,1,1)$ for $\sigma_{i}, \bar{\sigma}_{i}$, and $(1,0,0)$ for $\chi_{i}$ and $\bar{\chi}_{i}$ may then be obtained in a natural symmetry limit, for which the mismatch between the charged-lepton and neutrino mass matrices will exhibit tribimaximal mixing.

The most general superpotential of the singlet superfields invariant under $\Sigma(81)$ is given by

$$
\begin{aligned}
W & =m_{\sigma}\left(\sigma_{1} \bar{\sigma}_{1}+\sigma_{2} \bar{\sigma}_{2}+\sigma_{3} \bar{\sigma}_{3}\right)+m_{\chi}\left(\chi_{1} \bar{\chi}_{1}+\chi_{2} \bar{\chi}_{2}+\chi_{3} \bar{\chi}_{3}\right) \\
& +\frac{1}{3} f\left(\sigma_{1}^{3}+\sigma_{2}^{3}+\sigma_{3}^{3}\right)+\frac{1}{3} \bar{f}\left(\bar{\sigma}_{1}^{3}+\bar{\sigma}_{2}^{3}+\bar{\sigma}_{3}^{3}\right)+\frac{1}{3} h\left(\chi_{1}^{3}+\chi_{2}^{3}+\chi_{3}^{3}\right)+\frac{1}{3} \bar{h}\left(\bar{\chi}_{1}^{3}+\bar{\chi}_{2}^{3}+\bar{\chi}_{3}^{3}\right) \\
& +\lambda\left(\chi_{1} \sigma_{2} \sigma_{3}+\chi_{2} \sigma_{3} \sigma_{1}+\chi_{3} \sigma_{1} \sigma_{2}\right)+\bar{\lambda}\left(\bar{\chi}_{1} \bar{\sigma}_{2} \bar{\sigma}_{3}+\bar{\chi}_{2} \bar{\sigma}_{3} \bar{\sigma}_{1}+\bar{\chi}_{3} \bar{\sigma}_{1} \bar{\sigma}_{2}\right) .
\end{aligned}
$$

The resulting scalar potential has a supersymmetric minimum $(V=0)$ if

$$
0=m_{\sigma} \bar{\sigma}_{1}+f \sigma_{1}^{2}+\lambda\left(\chi_{2} \sigma_{3}+\chi_{3} \sigma_{2}\right)=m_{\sigma} \sigma_{1}+\bar{f} \bar{\sigma}_{1}^{2}+\bar{\lambda}\left(\bar{\chi}_{2} \bar{\sigma}_{3}+\bar{\chi}_{3} \bar{\sigma}_{2}\right)
$$




$$
\begin{aligned}
0 & =m_{\sigma} \bar{\sigma}_{2}+f \sigma_{2}^{2}+\lambda\left(\chi_{3} \sigma_{1}+\chi_{1} \sigma_{3}\right)=m_{\sigma} \sigma_{2}+\bar{f} \bar{\sigma}_{2}^{2}+\bar{\lambda}\left(\bar{\chi}_{3} \bar{\sigma}_{1}+\bar{\chi}_{1} \bar{\sigma}_{3}\right), \\
0 & =m_{\sigma} \bar{\sigma}_{3}+f \sigma_{3}^{2}+\lambda\left(\chi_{1} \sigma_{2}+\chi_{2} \sigma_{1}\right)=m_{\sigma} \sigma_{3}+\bar{f} \bar{\sigma}_{3}^{2}+\bar{\lambda}\left(\bar{\chi}_{1} \bar{\sigma}_{2}+\bar{\chi}_{2} \bar{\sigma}_{1}\right), \\
0 & =m_{\chi} \bar{\chi}_{1}+h \chi_{1}^{2}+\lambda \sigma_{2} \sigma_{3}=m_{\chi} \chi_{1}+\bar{h} \bar{\chi}_{1}^{2}+\bar{\lambda} \bar{\sigma}_{2} \bar{\sigma}_{3}, \\
0 & =m_{\chi} \bar{\chi}_{2}+h \chi_{2}^{2}+\lambda \sigma_{3} \sigma_{1}=m_{\chi} \chi_{2}+\bar{h} \bar{\chi}_{2}^{2}+\bar{\lambda} \bar{\sigma}_{3} \bar{\sigma}_{1}, \\
0 & =m_{\chi} \bar{\chi}_{3}+h \chi_{3}^{2}+\lambda \sigma_{1} \sigma_{2}=m_{\chi} \chi_{3}+\bar{h} \bar{\chi}_{3}^{2}+\bar{\lambda} \bar{\sigma}_{1} \bar{\sigma}_{2} .
\end{aligned}
$$

In the limit $\lambda=\bar{\lambda}=0$, the symmetry of $W$ is enlarged to $\Sigma(81) \times \Sigma(81)$. Thus it is natural to expect $\lambda, \bar{\lambda}<<f, \bar{f}, h, \bar{h}$, and as a first approximation, a possible solution of $V=0$ is

$$
\begin{aligned}
& \left\langle\sigma_{1,2,3}\right\rangle_{0}=-m_{\sigma}\left(f^{2} \bar{f}\right)^{-1 / 3}, \quad\left\langle\bar{\sigma}_{1,2,3}\right\rangle_{0}=-m_{\sigma}\left(\bar{f}^{2} f\right)^{-1 / 3}, \\
& \left\langle\chi_{1}\right\rangle_{0}=-m_{\chi}\left(h^{2} \bar{h}\right)^{-1 / 3}, \quad\left\langle\bar{\chi}_{1}\right\rangle_{0}=-m_{\chi}\left(\bar{h}^{2} h\right)^{-1 / 3}, \quad\left\langle\chi_{2,3}\right\rangle_{0}=\left\langle\bar{\chi}_{2,3}\right\rangle_{0}=0,
\end{aligned}
$$

where $\lambda=\bar{\lambda}=0$ has been assumed. [The appearance of domain walls can be avoided by explicit soft supersymmetry breaking terms which also break $\Sigma(81)$.] This results in the desirable Yukawa terms $\left(l_{1}+l_{2}+l_{3}\right) l_{1}^{c} \phi^{0},\left(l_{1}+\omega^{2} l_{2}+\omega l_{3}\right) l_{2}^{c} \phi^{0},\left(l_{1}+\omega l_{2}+\omega^{2} l_{3}\right) l_{3}^{c} \phi^{0}$, $\left(\nu_{1} \nu_{1}+\nu_{2} \nu_{2}+\nu_{3} \nu_{3}\right) \xi^{0}$, and $\nu_{2} \nu_{3} \xi^{0}$, leading to tribimaximal mixing [5]. Specifically

$$
\mathcal{M}_{l}=\left(\begin{array}{ccc}
h_{e} & h_{\mu} & h_{\tau} \\
h_{e} & \omega^{2} h_{\mu} & \omega h_{\tau} \\
h_{e} & \omega h_{\mu} & \omega^{2} h_{\tau}
\end{array}\right) v=\frac{1}{\sqrt{3}}\left(\begin{array}{ccc}
1 & 1 & 1 \\
1 & \omega^{2} & \omega \\
1 & \omega & \omega^{2}
\end{array}\right)\left(\begin{array}{ccc}
h_{e} & 0 & 0 \\
0 & h_{\mu} & 0 \\
0 & 0 & h_{\tau}
\end{array}\right) \sqrt{3} v
$$

and [6, 10]

$$
\mathcal{M}_{\nu}=\left(\begin{array}{lll}
a & 0 & 0 \\
0 & a & d \\
0 & d & a
\end{array}\right)=\left(\begin{array}{ccc}
0 & 1 & 0 \\
1 / \sqrt{2} & 0 & i / \sqrt{2} \\
1 / \sqrt{2} & 0 & -i / \sqrt{2}
\end{array}\right)\left(\begin{array}{ccc}
a+d & 0 & 0 \\
0 & a & 0 \\
0 & 0 & -a+d
\end{array}\right)\left(\begin{array}{ccc}
0 & 1 / \sqrt{2} & 1 / \sqrt{2} \\
1 & 0 & 0 \\
0 & i / \sqrt{2} & -i / \sqrt{2}
\end{array}\right),
$$

thereby leading to Eq. (1), i.e.

$$
\frac{1}{\sqrt{3}}\left(\begin{array}{ccc}
1 & 1 & 1 \\
1 & \omega & \omega^{2} \\
1 & \omega^{2} & \omega
\end{array}\right)\left(\begin{array}{ccc}
0 & 1 & 0 \\
1 / \sqrt{2} & 0 & i / \sqrt{2} \\
1 / \sqrt{2} & 0 & -i / \sqrt{2}
\end{array}\right)=\left(\begin{array}{ccc}
\sqrt{2 / 3} & \sqrt{1 / 3} & 0 \\
-\sqrt{1 / 6} & \sqrt{1 / 3} & -\sqrt{1 / 2} \\
-\sqrt{1 / 6} & \sqrt{1 / 3} & \sqrt{1 / 2}
\end{array}\right) .
$$

This is thus another version of a successful derivation of tribimaximal mixing, but as in all previous such models, the predicted value of $\tan ^{2} \theta_{12}=0.5$ is not the central value of 
present experimental data: $\tan ^{2} \theta_{12}=0.45 \pm 0.05$. To obtain a deviation from $\tan ^{2} \theta_{12}=0.5$ in the context of $\Sigma(81)$ alone, consider now $\lambda, \bar{\lambda} \neq 0$ but small. In that case,

$$
\begin{gathered}
\frac{\left\langle\chi_{2,3}\right\rangle}{\left\langle\chi_{1}\right\rangle_{0}} \simeq \frac{\bar{\lambda}\left(h^{2} \bar{h}\right)^{1 / 3}}{\left(f^{2} \bar{f}\right)^{2 / 3}} \frac{m_{\sigma}^{2}}{m_{\chi}^{2}}, \quad \frac{\left\langle\bar{\chi}_{2,3}\right\rangle}{\left\langle\bar{\chi}_{1}\right\rangle_{0}} \simeq \frac{\lambda\left(\bar{h}^{2} h\right)^{1 / 3}}{\left(\bar{f}^{2} f\right)^{2 / 3}} \frac{m_{\sigma}^{2}}{m_{\chi}^{2}} \\
\delta\left\langle\sigma_{1}\right\rangle \simeq 0, \quad \frac{\delta\left\langle\sigma_{2,3}\right\rangle}{\langle\sigma\rangle_{0}} \simeq-\frac{m_{\chi}}{3 m_{\sigma}}\left[\frac{\bar{\lambda} f^{1 / 3}}{\left(\bar{h}^{2} h \bar{f}\right)^{1 / 3}}+\frac{2 \lambda \bar{f}^{1 / 3}}{\left(h^{2} \bar{h} f\right)^{1 / 3}}\right], \\
\delta\left\langle\bar{\sigma}_{1}\right\rangle \simeq 0, \quad \frac{\delta\left\langle\bar{\sigma}_{2,3}\right\rangle}{\langle\bar{\sigma}\rangle_{0}} \simeq-\frac{m_{\chi}}{3 m_{\sigma}}\left[\frac{\lambda \bar{f})^{1 / 3}}{\left(h^{2} \bar{h} f\right)^{1 / 3}}+\frac{2 \bar{\lambda} f^{1 / 3}}{\left(\bar{h}^{2} h \bar{f}\right)^{1 / 3}}\right] .
\end{gathered}
$$

Since $\left\langle\bar{\sigma}_{1}\right\rangle \neq\left\langle\bar{\sigma}_{2,3}\right\rangle$, the charged-lepton mass matrix is modified. Instead of Eq. (17), it is now of the form

$$
\mathcal{M}_{l}=\left(\begin{array}{ccc}
h_{e} v_{1} & h_{\mu} v_{1} & h_{\tau} v_{1} \\
h_{e} v_{2} & \omega^{2} h_{\mu} v_{2} & \omega h_{\tau} v_{2} \\
h_{e} v_{2} & \omega h_{\mu} v_{2} & \omega^{2} h_{\tau} v_{2}
\end{array}\right) .
$$

Using the phenomenological hierarchy $h_{e}<<h_{\mu}<<h_{\tau}$, it is easily shown [15], to first approximation, that the tribimaximal $U_{\alpha i}$ of Eq. (1) is multiplied on the left by

$$
R=\left(\begin{array}{ccc}
1 & -r & -r \\
r & 1 & -r \\
r & r & 1
\end{array}\right), \quad r \simeq \frac{v_{1}-v_{2}}{v_{1}+2 v_{2}} \simeq-\frac{\delta\left\langle\bar{\sigma}_{2,3}\right\rangle}{3\langle\bar{\sigma}\rangle_{0}} .
$$

The neutrino mass matrix of Eq. (18) is also changed, i.e.

$$
\mathcal{M}_{\nu}=\left(\begin{array}{ccc}
a & e & e \\
e & a+b & d \\
e & d & a+b
\end{array}\right)
$$

where $|b|<<|a|$ and $|e|<<|d|$. This leads to a correction of Eq. (1) on the right by the matrix

$$
R^{\prime}=\left(\begin{array}{ccc}
1 & -r^{\prime} & 0 \\
r^{\prime} & 1 & 0 \\
0 & 0 & 1
\end{array}\right), \quad r^{\prime} \simeq \frac{\sqrt{2} e}{d} \simeq \frac{\sqrt{2}\left\langle\chi_{2,3}\right\rangle}{\left\langle\chi_{1}\right\rangle_{0}} .
$$

Hence the corrected mixing matrix is given by

$$
U_{\alpha i} \simeq\left(\begin{array}{ccc}
\sqrt{2 / 3}\left(1+r+r^{\prime} / \sqrt{2}\right) & \sqrt{1 / 3}\left(1-2 r-\sqrt{2} r^{\prime}\right) & 0 \\
-\sqrt{1 / 6}\left(1-3 r-\sqrt{2} r^{\prime}\right) & \sqrt{1 / 3}\left(1+r^{\prime} / \sqrt{2}\right) & -\sqrt{1 / 2}(1+r) \\
-\sqrt{1 / 6}\left(1-r-\sqrt{2} r^{\prime}\right) & \sqrt{1 / 3}\left(1+2 r+r^{\prime} / \sqrt{2}\right) & \sqrt{1 / 2}(1-r)
\end{array}\right) .
$$


Therefore,

$$
\tan ^{2} \theta_{12} \simeq \frac{1}{2}-3\left(r+r^{\prime} / \sqrt{2}\right), \quad \tan ^{2} \theta_{23} \simeq 1+4 r, \quad \theta_{13} \simeq 0 .
$$

For example, let $r=r^{\prime}=0.01$, then $\tan ^{2} \theta_{12} \simeq 0.45$, whereas $\tan ^{2} \theta_{23} \simeq 1.04$ which is equivalent to $\sin ^{2} 2 \theta_{23} \simeq 0.9996$. A better match to the data is thus obtained.

In summary, it has been shown in this paper that neutrino tribimaximal mixing is a natural limit in a supersymmetric model based on $\Sigma(81)$ without any imposed auxiliary symmetry as in previous models. The assumed particle content and the requirement of supersymmetry allow a prediction (tribimaximal mixing) in the limit that the superpotential has the enlarged symmetry $\Sigma(81) \times \Sigma(81)$. The parameters which break $\Sigma(81) \times \Sigma(81)$ but preserve $\Sigma(81)$ are assumed to be small to check the possible deviation from tribimaximal mixing. The conclusions are that whereas corrections are expected, they are such that only $\tan ^{2} \theta_{12}$ may deviate significantly from 0.5 (say to 0.45 ) without affecting much the predictions $\sin ^{2} 2 \theta_{23}=1$ and $\theta_{13}=0$. [It should be pointed out that in many models, the $a d$ hoc assumption $\nu_{2}=\left(\nu_{e}+\nu_{\mu}+\nu_{\tau}\right) / \sqrt{3}$ is made. In that case, $\tan ^{2} \theta_{12}$ must be greater than 0.5 , not smaller.]

This work was supported in part by the U. S. Department of Energy under Grant No. DEFG03-94ER40837.

\section{Appendix}

The 9 one-dimensional irreducible representations together with $\mathbf{3}_{\mathbf{D}}, \overline{\mathbf{3}}_{\mathbf{D}}$ behave as in $\Delta(27)$, i.e. 21

$$
\mathbf{3}_{\mathrm{D}} \times \mathbf{3}_{\mathrm{D}}=\overline{\mathbf{3}}_{\mathrm{D}}+\overline{\mathbf{3}}_{\mathrm{D}}+\overline{\mathbf{3}}_{\mathrm{D}}, \quad \mathbf{3}_{\mathrm{D}} \times \overline{\mathbf{3}}_{\mathrm{D}}=\mathbf{1}_{1,2,3}+\mathbf{1}_{\mathbf{4}, 5,6}+\mathbf{1}_{7,8,9}
$$

The $\mathbf{3}_{\mathbf{A}}, \mathbf{3}_{\mathbf{B}}, \mathbf{3}_{\mathrm{C}}$ representations are cyclically equivalent, as are their conjugates. Their multiplication rules are

$$
\mathbf{3}_{\mathrm{A}} \times \overline{\mathbf{3}}_{\mathrm{A}}=\mathbf{3}_{\mathrm{B}} \times \overline{3}_{\mathrm{B}}=\mathbf{3}_{\mathrm{C}} \times \overline{3}_{\mathrm{C}}=\mathbf{1}_{1,2,3}+\mathbf{3}_{\mathrm{D}}+\overline{3}_{\mathrm{D}}
$$




$$
\begin{aligned}
& 3_{\mathrm{B}} \times \overline{3}_{\mathrm{A}}=3_{\mathrm{C}} \times \overline{3}_{\mathrm{B}}=3_{\mathrm{A}} \times \overline{3}_{\mathrm{C}}=\mathbf{1}_{4,5,6}+3_{\mathrm{D}}+\overline{3}_{\mathrm{D}} \\
& 3_{\mathrm{C}} \times \overline{3}_{\mathrm{A}}=3_{\mathrm{A}} \times \overline{3}_{\mathrm{B}}=3_{\mathrm{B}} \times \overline{3}_{\mathrm{C}}=\mathbf{1}_{7,8,9}+3_{\mathrm{D}}+\overline{3}_{\mathrm{D}} \\
& 3_{\mathrm{A}} \times 3_{\mathrm{A}}=3_{\mathrm{B}} \times \mathbf{3}_{\mathrm{C}}=\overline{3}_{\mathrm{A}}+\overline{3}_{\mathrm{B}}+\overline{3}_{\mathrm{B}}, \\
& 3_{\mathrm{B}} \times 3_{\mathrm{B}}=3_{\mathrm{C}} \times 3_{\mathrm{A}}=\overline{3}_{\mathrm{B}}+\overline{3}_{\mathrm{C}}+\overline{3}_{\mathrm{C}}, \\
& 3_{\mathrm{C}} \times 3_{\mathrm{C}}=3_{\mathrm{A}} \times 3_{\mathrm{B}}=\overline{3}_{\mathrm{C}}+\overline{3}_{\mathrm{A}}+\overline{3}_{\mathrm{A}} .
\end{aligned}
$$

\section{References}

[1] See for example T. Schwetz, Phys. Scripta T127, 1 (2006).

[2] P. F. Harrison, D. H. Perkins, and W. G. Scott, Phys. Lett. B530, 167 (2002).

[3] E. Ma and G. Rajasekaran, Phys. Rev. D64, 113012 (2001).

[4] K. S. Babu, E. Ma, and J. W. F. Valle, Phys. Lett. B552, 207 (2003).

[5] E. Ma, Phys. Rev. D70, 031901R (2004).

[6] G. Altarelli and F. Feruglio, Nucl. Phys. B720, 64 (2005).

[7] E. Ma, Phys. Rev. D72, 037301 (2005).

[8] K. S. Babu and X.-G. He, hep-ph/0507217.

[9] A. Zee, Phys. Lett. B630, 58 (2005).

[10] G. Altarelli and F. Feruglio, Nucl. Phys. B741, 215 (2006).

[11] E. Ma, Phys. Rev. D73, 057304 (2006).

[12] X.-G. He, Y.-Y. Keum, and R. R. Volkas, JHEP 0604, 039 (2006).

[13] G. Altarelli, F. Feruglio, and Y. Lin, Nucl. Phys. B775, 31 (2007). 
[14] S. F. King and M. Malinsky, Phys. Lett. B645, 351 (2007).

[15] E. Ma, Mod. Phys. Lett. A22, 101 (2007).

[16] X.-G. He, Nucl. Phys. Proc. Suppl. 168, 350 (2007).

[17] E. Ma, Phys. Lett. B649, 287 (2007).

[18] E. Ma, Mod. Phys. Lett. A17, 2361 (2002).

[19] G. C. Branco, J.-M. Gerard, and W. Grimus, Phys. Lett. B136, 383 (1984).

[20] I. de Medeiros Varzielas, S. F. King, and G. G. Ross, Phys. Lett. B648, 201 (2007).

[21] E. Ma, Mod. Phys. Lett. A21, 1917 (2006). 